\title{
Protocol Enhancements in LEACH
}

\author{
Neha Aggarwal \\ Electronics \& Communication, IIMT Meerut \\ Meerut, India
}

\begin{abstract}
LEACH is a hierarchical protocol in which most nodes transmit to cluster heads, and the cluster heads aggregate and compress the data and forward it to the base station (sink).In LEACH, a TDMA-based MAC protocol is integrated with clustering and a simple "routing" protocol. The goal of LEACH is to lower the energy consumption required to create and maintain clusters or to use the energy of the nodes in such a manner so as to improve the life time of a wireless sensor network. In this paper we are presenting an overview of the different protocol changes made in LEACH to improve network lifetime, throughput, coverage area of network etc.
\end{abstract}

Keywords-LEACH, E-LEACH, Enhanced LEACH, EHE-LEACH, Multi-level Leach

\section{LEACH: PROTOCOL EXPANATION}

LEACH, Low-energy Adaptive Clustering Hierarchy, presented by Wendi B. Heinzelman of MIT, is application specific protocol architecture for Wireless Sensor Network (WSN). It assumes a dense sensor network of homogeneous, energy-constrained nodes, which shall report their data to a sink node. It has become one of the most used bases for developing new architecture protocols or modifying the existing one in WSN.

LEACH [6] is a TDMA-based MAC protocol which is integrated with clustering and a simple routing protocol in wireless sensor network. LEACH partitions the nodes into clusters and in each cluster a dedicated node, the clusterhead, is responsible for creating and maintaining a TDMA schedule; all the other nodes of a cluster are member nodes. To all member nodes, TDMA slots are assigned, which can be used to exchange data between the member and the clusterhead; there is no peer-to-peer communication. Except their time slots, the members can spend their time in sleep state. The clusterhead aggregates the data and transmits it to the sink. Since the sink is often far away, the clusterhead must spend significant energy for this transmission. For a member, it is typically much cheaper to reach the clusterhead than to transmit directly to the sink. The clusterheads role is energy consuming since it is always switched on and is responsible for the long-range transmissions. If a fixed node has this role, it would burn its energy quickly, and after it died, all its members would be "headless" and therefore useless. Therefore, this burden is rotated among the nodes.

Specifically, each node decides independent of other nodes whether it becomes a clusterhead, and therefore there is no signalling traffic related to clusterhead election (although signalling traffic is needed for subsequent association of nodes to some clusterhead). This decision takes into account when the node served as clusterhead the last time, such that a node that has not been a clusterhead for a long time is more likely to elect itself than a node serving just recently. The protocol is round based, that is, all nodes make their decisions whether to become a clusterhead at the same time and the nonclusterhead nodes have to associate to a clusterhead subsequently. The nonclusterheads choose their clusterhead based on received signal strengths. The network partitioning into clusters is time variable and the protocol assume global time synchronization.

After the clusters have been formed, each clusterhead picks a random CDMA code for its cluster, which it broadcasts and which its member nodes have to use subsequently. This avoids a situation where a border node belonging to clusterhead $A$ distorts transmissions directed to clusterhead $B$, shown in Figure1.

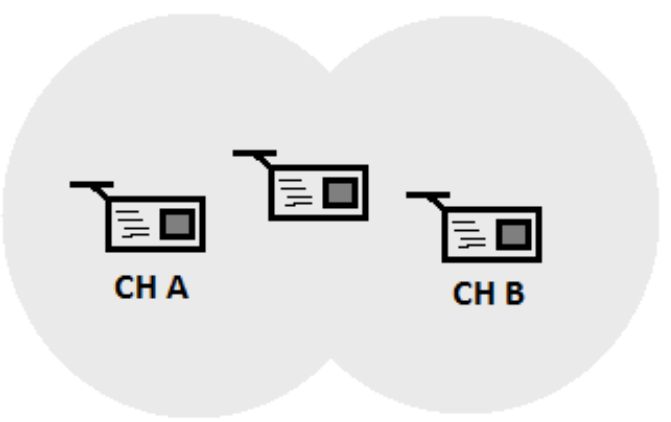

Fig 1

A critical network parameter is the percentage of nodes that are clusterheads. If there are only a few clusterheads, the expected distance between a member node and its clusterhead becomes longer and therefore the member has to spend more energy to reach its clusterhead while maintaining a given BER target. On the other hand, if there are many clusterheads, there will be more energy expensive transmissions from clusterheads to the sink and less aggregation. Therefore, there exists an optimum percentage of clusterheads, which for the scenario investigated in is $\approx 5 \%$. If this optimum is chosen, LEACH can achieve a seven to eight times lower overall energy dissipation compared to the case where each node transmits its data directly to the sink, and between four and eight times lower energy than in a scenario where packets are relayed in a multi-hop fashion. In addition, since LEACH distributes the clusterhead role fairly to all nodes, they tend to die at about the same time.

The protocol is organized in rounds and each round is subdivided into a setup phase and a steady-state phase, Figure2. The setup phase starts with the self-election of nodes to clusterheads. 
In the following advertisement phase, the clusterheads inform their neighborhood with an advertisement packet. The clusterheads contend for the medium using a CSMA protocol with no further provision against the hidden-terminal problem.

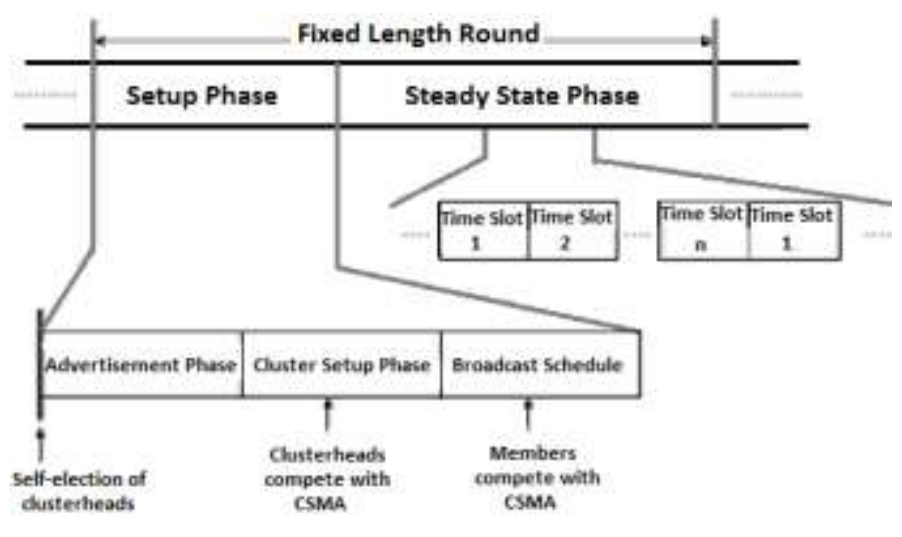

Fig 2

The nonclusterhead nodes pick the advertisement packet with the strongest received signal strength. In the following clustersetup phase, the members inform their clusterhead ("join"), again using a CSMA protocol. After the cluster setup-phase, the clusterhead knows the number of members and their identifiers. It constructs a TDMA schedule, picks a CDMA code randomly, and broadcasts this information in the broadcast schedule sub phase. After this, the TDMA steadystate phase begins. Because of collisions of advertisement or join packets, the protocol cannot guarantee that each nonclusterhead node belongs to a cluster. However, it can guarantee that nodes belong to at most one cluster as shown in Fig 3 .

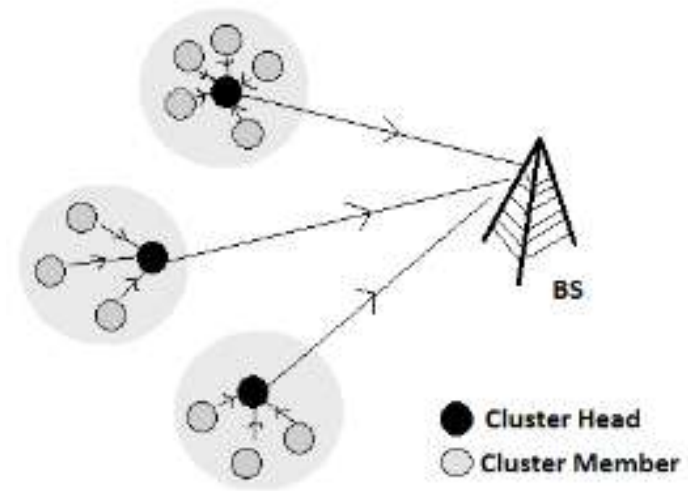

Fig 3

The clusterhead is switched on during the whole round and the member nodes have to be switched on during the setup phase and occasionally in the steady-state phase, according to their position in the cluster's TDMA schedule.

Nodes that have been cluster heads cannot become cluster heads again for $P$ rounds, where $P$ is the desired percentage of cluster heads. Thereafter, each node has a $1 / P$ probability of becoming a cluster head in each round.

In the setup phase, each node decides whether or not to become a cluster head for current round. The selection depends on decision made by the node by choosing a random number between 0 and 1 . If the number is less than the threshold T(n), the node becomes a clusterhead for the current round. The threshold is set as:

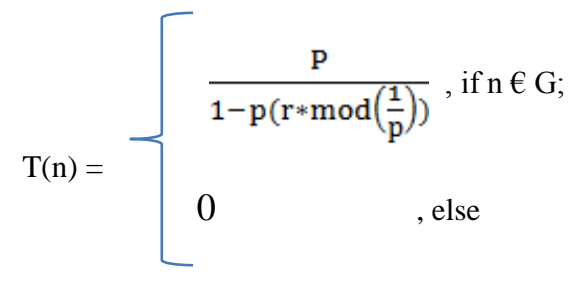

Where $\mathrm{p}$ is the probability of the node being selected as a cluster-head node, $r$ is the number of rounds passed, and $\mathrm{G}$ is the set of nodes that have not been cluster-heads in the last $1 / \mathrm{p}$ rounds, mod denotes modulo operator. Nodes that are cluster heads in round $r$ shall not be selected in the next $1 / p$ rounds. The random selection algorithm for LEACH easily leads to the imbalance of the stored energy of sensor nodes, thereby; the consumption of system energy is increased.

\section{Enhanced LEACH}

In LEACH protocol, time is divided into many rounds, in each round, all the nodes contend to be cluster head according to a predefined criterion. Enhanced LEACH [1] protocol was proposed to set the time length of each round, to prolong the lifetime of the network and increase throughput, which is denoted as the amount of data packs sent to the sink node. LEACH protocol lets the data transmission phase last for a fixed period of time, then enter into a new round of cluster head election. The time length of round has obviously influence on the performance of LEACH protocol. In order to decrease the overhead of set-up phase, we hope to increase the time length of round, which increases the time for data transmission. However, prolonging the time length of round also increases the energy consumption of cluster head, which will causes some nodes die early and in turn shortens the lifetime of wireless sensor networks. So, regarding to configuration of time length of round, there is a trade-off between lifetime and throughput.

If the time of set-up phase is $\alpha$, and the steady data transmission time is $t$, then the time length of every round is $t_{r}$ $=\alpha+\mathrm{t}$. Let the time when the first sensor node dies as the lifetime of the network, which is denoted as $t_{\text {fnd }}$.

$\mathrm{t}_{\mathrm{fnd}}=\mathrm{n}(\alpha+\mathrm{t})$, Where $\mathrm{n}$ is the number of rounds after which the first sensor node dies.

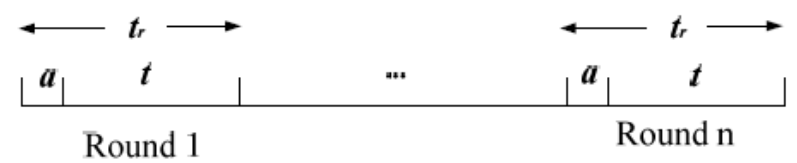

Fig 4

According to LEACH protocol, there are $\mathrm{m}$ frames in the time $t$, so frame $t=m \cdot T$, here frame $T$ is the time length of each frame. As the time length of a round increase with $\mathrm{m}$, increasing $\mathrm{m}$ will increase the energy consumption of cluster head in a round, which makes the energy consumption in the network unbalanced. Therefore, some nodes will die early and 
in turn shortens the lifetime of wireless sensor networks. So as per Fig 5 and Fig $6, \mathrm{~m}=5$ is good for trade-off between lifetime and throughput.

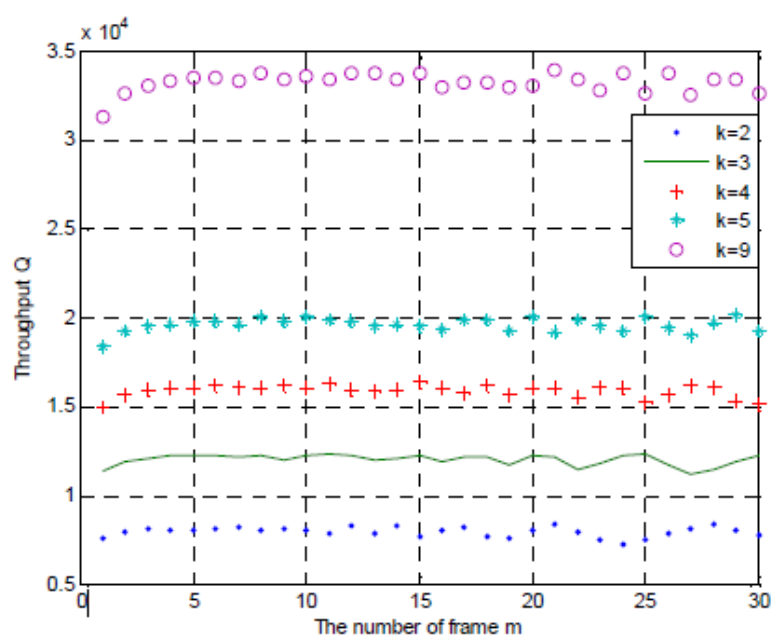

Fig 5

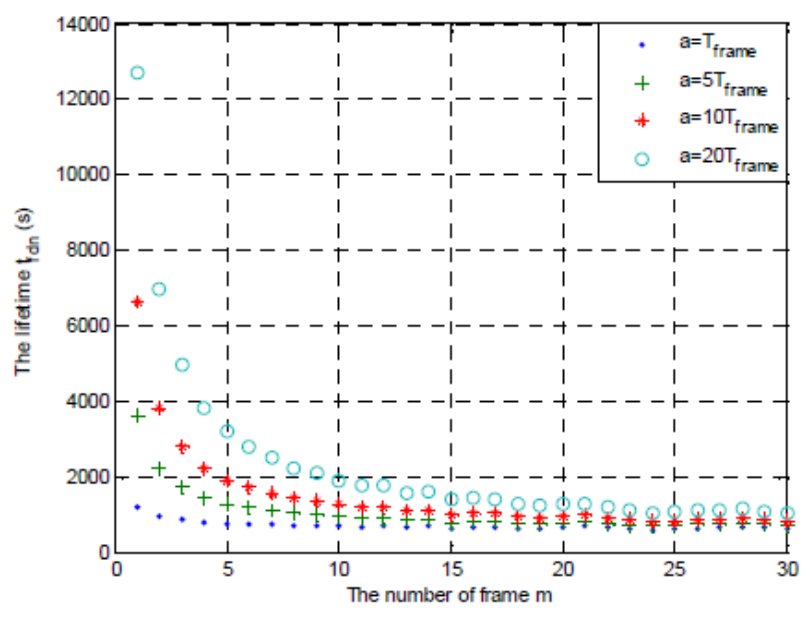

Fig 6

\section{E-LEACH}

In the E-LEACH [2] algorithm, the original way of the selection of the cluster heads is random and the round time for the selection is fixed. The nodes with low remnant energy have the same priority to be a cluster head as the node with high remnant energy. Therefore, those nodes with less remaining energy may be chosen as the cluster heads which will result that these nodes may die first. In the E-LEACH algorithm, we consider the residual power of the sensor nodes in order to balance network loads and changes the round time depending on the optimal cluster size.

The cluster heads communicate with the base station in single-hop mode which makes LEACH inefficient to be used in large-scale wireless sensor networks for the limit effective communication range of the sensor nodes. The E-LEACH adopts the same round concept with the original LEACH. In hierarchical routing protocols, the number of cluster-heads is a key factor that affects the performance of routing protocols. If the number of cluster-heads is less, each cluster-head needs to cover larger region, this will lead the problem that some cluster-members get far from their cluster-heads and consume much more energy. As the communication between cluster heads and the base station needs much more energy than common nodes, the excessive number of cluster-heads will increase the energy consumption of the whole network and shorten the network lifetime. Therefore, it is necessary to select optimal clusterhead number to make the energy consumption minimum. In the E-LEACH we use the minimum spanning tree between clusterheads, choose the cluster head which has largest residual energy as the root node. Fig 7 depicts the architecture of ELEACH.

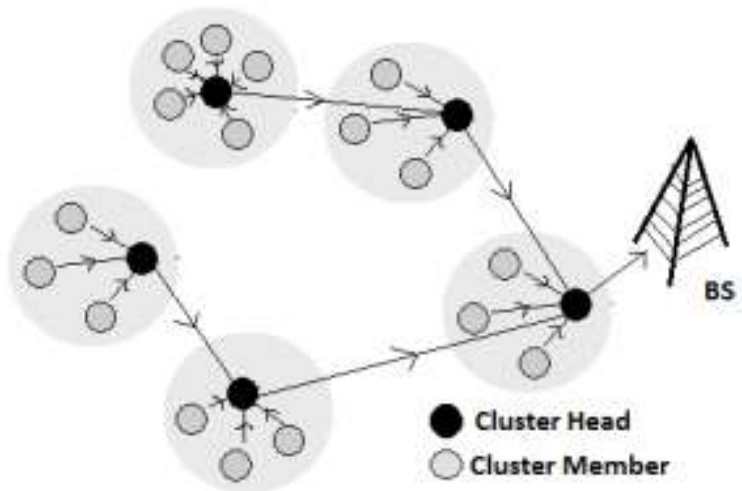

Fig 7

Fig. 8 is the comparison of FND and HND between LEACH and E-LEACH. If the system lifetime is defined as the number of rounds for which the first node dies, E-LEACH algorithm can reach 1529 rounds, whereas LEACH only reaches 1029 rounds. The system lifetime is improved by $49 \%$ of ELEACH algorithm than LEACH.

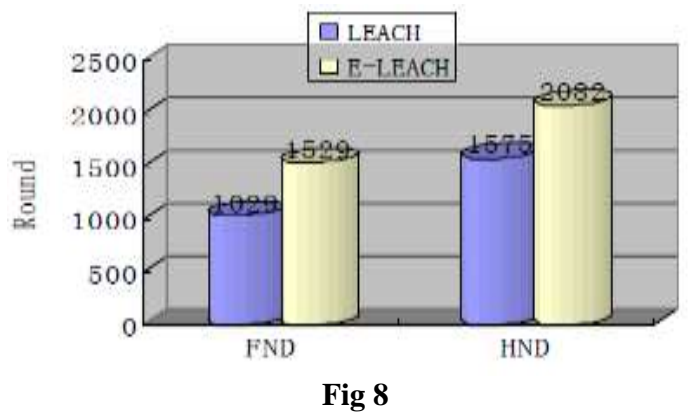

The simulation results show that our proposed protocol increases network lifetime at least by $40 \%$ when compared with the LEACH algorithm.

\section{EHE-LEACH}

The major problem of the sensor node is the limited energy. Therefore, energy efficiency in the design of routing protocols for sensor networks is of principal requirement. EHE-LEACH [3] (Enhanced HEterogeneous - Low Energy Adaptive Cluster based Hierarchical protocol) is based on the fixed distance threshold, which is used to bifurcate the coverage area, used two separate mechanism direct diffusion and clustering. From a survey it is observed that minimum two hop data transmission is required for the cluster based communication, from source to BS even though the distance between source 
and BS is very small. This consumes extra execution time for the completion of a round.

From Fig. 9 it is clear that $\mathrm{d} 3>\mathrm{d} 2>\mathrm{d} 1$. For LEACH protocol single hop communication is not, minimum two hope communication is required.

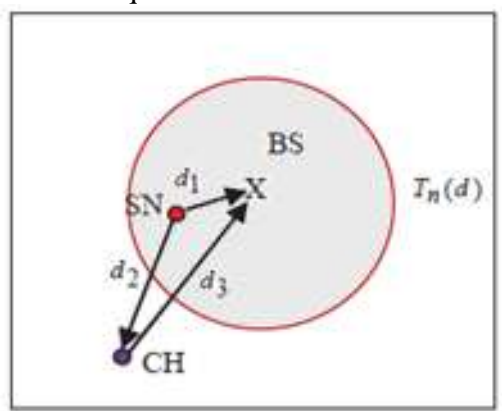

Fig 9

In the proposed model two parameters are considered: firstlyminimize the execution time of a round to some extent by using direct diffusion. Secondly- maximizes the lifetime and stability of the network by using the combination of two techniques simultaneously direct diffusion and clustering. The half nodes alive (HNA) \& last node alive (LNA) are the key parameters used for the measurement of lifetime and the stability of the system. SNs near to the BS communicate directly and those which are far away from the BS use cluster based communication. To evaluate the performance of the proposed scheme two key parameters known as: Half Nodes Alive (HNA) and Last Node Alive (LNA) are selected. By selecting the distance based threshold with the ratio of 1:9 between direct communication and cluster based communication it has been observed that EHE-LEACH has better network lifetime with respect to various parameters in comparison to the other well-known proposals such as LEACH.

\section{Multi-Level LEACH}

Although LEACH protocol conserves energy in the nodes and prolongs the lifetime of the WSN application, it still has the following problems:

- Randomized rotation of the $\mathrm{CH}$ without considering the residual energy in the nodes.

- As the network size increases the CH's which are far away from the BS drain their energy sooner.

If the deployment area is less, the LEACH protocol works well. Since the LEACH protocol cannot be used over large areas, a Hierarchical routing protocol based on the LEACH.

In Hierarchical routing protocol [4] the $\mathrm{CHs}$ are divided into various levels so that each

$\mathrm{CH}$ at a level i transmits the data to its higher level, $(\mathrm{i}+1)$ level $\mathrm{CH}$, instead of transmitting the data to the BS directly. Two-level LEACH (TL-LEACH), Three-level LEACH (3LLEACH), Four-level LEACH (4L-LEACH) can be implemented to install multi-level LEACH.

\subsection{TL-LEACH}

In this, we have a two- level structure consisting of leaf nodes, level-1 CH's, level-2 CH's. One of the level-1 CH's is chosen as level-2 $\mathrm{CH}$ as shown in figure 3 . After collecting the data, a level-1 $\mathrm{CH}$ forwards the same to its level-2 $\mathrm{CH}$. This level-2 $\mathrm{CH}$ aggregates the data received from its level-1 $\mathrm{CH}$ 's and the same aggregated data is forwarded to the BS resulting in energy savings. The operation TL-LEACH can be divided into the following 3 phases, namely:

- Selection of level-1 and level-2 CH's

- Set up phase

- Steady state phase

1) Selection of level-1 and level-2 CH's:

In this the level- $1 \mathrm{CH}$ is elected as given in the LEACH protocol. After the election of level-1 $\mathrm{CHs}$, the level-2 $\mathrm{CH}$ election is initiated. Among level- $1 \mathrm{CHs}$, the $\mathrm{CH}$ with maximum residual energy and proximate to the $\mathrm{BS}$ is elected as the level-2 $\mathrm{CH}$ as shown figure 3. In addition to its current role as level-1 $\mathrm{CH}$, the node acts as level-2 $\mathrm{CH}$ as well.

2) Set-up Phase:

After the election of level-1 $\mathrm{CHs}$, each $\mathrm{CH}$ broadcasts an advertisement message to all its leaf nodes. Each leaf node based on received signal strength from all the $\mathrm{CHs}$, decides which $\mathrm{CH}$ to join. Then each leaf node transmits an acknowledgement message to its chosen $\mathrm{CH}$ along with its node ID. Each level-1 CH records the node IDs of its member nodes. The set up phase for level-2 $\mathrm{CH}$ is also similar. In this case, level-1 CHs are treated as the leaf nodes. Each level-1 $\mathrm{CHs}$ sends a message containing the number of its member and its own node ID to the elected level-2 $\mathrm{CH}$. Thus, a level-2 $\mathrm{CH}$ has information regarding its level-1 $\mathrm{CHs}$ and their associated leaf nodes. So based on this information a level-2 $\mathrm{CH}$ creates a TDMA schedule to its members and broadcasts a message.

3) Steady state phase:

In the steady state phase transmission of the data takes place. Each leaf node sends the sensed data to its respective $\mathrm{CH}$ within its allocated time slot. Until their allocated time slot the leaf nodes can turn off their radio so that energy can be saved. All the leaf nodes transmit their data to its respective level-1 $\mathrm{CH}$. And the level-1 CHs aggregate all the sensed data. The aggregated data received by the level- $\mathrm{CH}$ is forwarded to their level-2 $\mathrm{CH}$ based on its allocated time slot. Thus the data collected by the level-2 $\mathrm{CH}$ is sent to the BS. Thus the data from a leaf node to the $\mathrm{BS}$ is transmitted using level-1 $\mathrm{CH}$ and then level-2 CH. Considerable energy in the WSN application can be saved with this type of hierarchical routing.

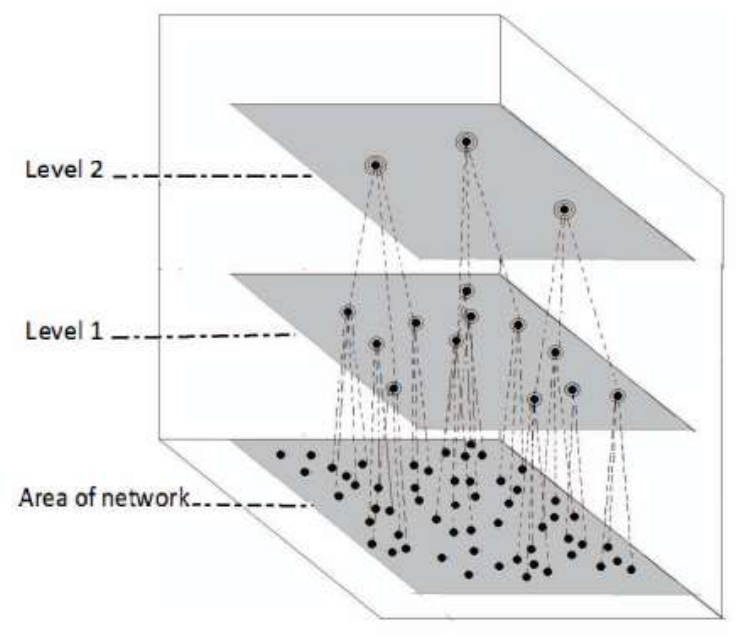

Fig 10

This work presents a multi-level hierarchical routing protocol, which is based on the LEACH protocol. This protocol improves both the energy efficiency and the lifetime of the network. Two-level LEACH (TL-LEACH), Three-level 
LEACH (3L-LEACH) and Four-level LEACH (4L-LEACH) have been presented. Analysis (done using NS3) shows that the hierarchical routing protocols, TL-LEACH, 3L-LEACH and 4L-LEACH, are better than the LEACH protocol.

It can be concluded that the multi-level Hierarchical routing protocol is more energy efficient when compared to the LEACH. The lifetime of the network also gets extended. As the deployment area of WSN increases, the 3L-LEACH and 4L-LEACH perform well when compared to the $2 \mathrm{~L}-\mathrm{LEACH}$.

\section{Op-LEACH}

LEACH uses a TDMA based MAC protocol, in order to maintain balanced energy consumption. The TDMA schedule maintained by Cluster Head evenly distributes the slots to all of the sensor nodes in its cluster. A number of these TDMA slots are wasted when the nodes have random data distribution. A modification to existing LEACH protocol is needed in order to use the slots corresponding to nodes that do not have data to send at its scheduled slot.

Op-LEACH [5] improves the performance of LEACH algorithm in terms of energy and time delay in real time networks. Every sensor node does not have data all the time. The data is available in random fashion. The sensors may be event driven, so data may be available only when they sense the event. The proposed method is utilizing the slots belonging to the node having no data to send. This method turns free slots into useful slots without making any changes in the TDMA schedule. This will reduce the waiting time for sensor nodes because now sensor nodes can get more than one slot per frame. It will reduce the data transmission delay and increase throughput of the network. The real time sensors are mostly event driven. So until the event occurs, the nodes will not have the data to process or to send (to the cluster head). So the corresponding slots become idle. The proposed algorithm utilizes these idle slots for some other node's data transmission. At the end of setup phase every non cluster head member knows about transmission schedule. Every node waits for its turn to transmit data and if a node does not have any data to transmit, the slot is allotted to the next sensor in TDMA schedule. This algorithm makes the free slots available to sensor nodes with higher traffic without additional overhead Op-LEACH shows effective performance difference when the data sensed by network nodes is uneven.

\section{CONCLUTION}

With the protocols discussed so far, it is clear that LEACH would not be able to cover large geographical areas of some square miles or more, because a clusterhead two miles away from the sink likely does not have enough energy to reach the sink at all. If clusterheads use other clusterheads for forwarding, this limitation can be mitigated. Even the slots are wasted in uneven traffic conditions which can be allotted to other nodes for forwarding. Energy efficiency is a key design issue in WSN and must be enhanced in order to improve network life time. Many such modifications can be made in LEACH to increase lifetime and throughput.

\section{REFERENCES}

[1] "Enhancing the Performance of LEACH Protocol in Wireless Sensor Network" IEEE INFOCOM 2011 Workshop on M2MCN-2011.

[2] "Improvement of LEACH protocol for WSN" 2012 9th International Conference on Fuzzy Systems and Knowledge Discovery (FSKD 2012).

[3] "EHE-LEACH: Enhanced heterogeneous LEACH protocol for lifetime enhancement of wireless SNs", 2013 International Conference on Advances in Computing, Communications and Informatics (ICACCI).

[4] Multi-level LEACH Protocol model using NS-3, 2014 IEEE International Advance Computing Conference (IACC).

[5] Op-LEACH: An Optimized LEACH Method for busty traffic in WSNs, 2014 Fourth International Conference on Advanced Computing \& Communication Technologies.

[6] Holger Karl and Andreas Willig "PROTOCOLS AND ARCHITECTURES FOR WIRELESS SENSOR NETWORKS", John Wiley \& Sons. 